\title{
On the aromaticity of uracil and its 5 -halogeno derivatives as revealed by theoretically derived geometric and magnetic indexes
}

\author{
Kacper Rzepiela ${ }^{1}$ (D) Aneta Buczek ${ }^{1}$ (D) $\cdot$ Teobald Kupka $^{1}$ (D) $\cdot$ Małgorzata A. Broda $^{1}$ (D)
}

Received: 8 September 2020 / Accepted: 13 November 2020 / Published online: 23 November 2020

(C) The Author(s) 2020

\begin{abstract}
The problem of aromaticity in heterocyclic rings of uracil and its 5 -halogenoderivatives (5XU) was analyzed theoretically by calculating modified harmonic oscillator model of aromaticity (HOMA) for Heterocycle Electron Delocalization (HOMHED), nucleus-independent chemical shift parameters (NICS) and the so-called scan experiments, using helium-3 atom as a magnetic probe. The impact of halogen electronegativity on C5 atom's NBO charges was also investigated. Water, as a polar environment, has a negligible impact on 5XU aromaticity. The most stable diketo tautomer shows a very low aromaticity while the "rare" dihydroxy form (tautomer No 6) is aromatic and resembles benzene. This is in agreement with traditional drawing of chemical formula of uracil's six-membered ring, directly showing three alternating single and double bonds in its tautomer No 6 . No good correlation between magnetic and geometric indexes of aromaticity for the studied 5XU tautomers was found. Linear correlation between the magnitude of NICS minimum, as well as the distance of the minimum above uracil ring plane center from ${ }^{3} \mathrm{He}$ NMR chemical shift scan plot with respect to halogen electronegativity were observed. A strong linear dependence of magnetic index of aromaticity and the electronegativity of $5 \mathrm{X}$ substituent was observed.
\end{abstract}

Keywords Uracil $\cdot 5$-halogenouracil $\cdot$ Aromaticity $\cdot$ HOMA $\cdot$ HOMHED $\cdot$ NICS $\cdot{ }^{3} \mathrm{He}$ NMR

\section{Introduction}

Uracil is an important component of living systems and belongs to small molecules which are responsible for information transfer [1]. Its structure could be derived from pyrimidine (1,3-diazine). Detection of the occurrence of uracil and pyrimidine in space [2] indirectly supports the fact that such molecules, containing two nitrogen atoms in a six-membered ring are stable and present in both extraterrestrial space matter and in living systems. For over 50 years, its 5-halogeno

Teobald Kupka

teobaldk@gmail.com

Małgorzata A. Broda

broda@uni.opole.pl

Kacper Rzepiela

119680@student.uni.opole.pl

Aneta Buczek

aneta.buczek@uni.opole.pl

1 Department of Physical Chemistry and Molecular Modeling, Faculty of Chemistry, University of Opole, 48 Oleska Street, 45-052 Opole, Poland derivatives have been studied [3,4]. Among them, 5fluorouracil has been widely used as an anticancer drug [3, 4].

The stability of uracil and its derivatives reflect their specific structure. Recently, on the basis of theoretical modeling using density functional theory (DFT at B3LYP-D3/aug-ccpVQZ level of theory), it was postulated [5] that mutual arrangement of local $\mathrm{C}=\mathrm{O}$ and $\mathrm{N}-\mathrm{H}$ functional groups in uracil, as well as in 1,3-diazines, influences the electron density and local dipole moments distribution in such a way that it is energetically more favorable than in both 1,2- and 1,4diazines.

Stability of many organic molecules is often related to aromaticity [6-10]. Among them are cyclic organic compounds, with double bonds and heteroatoms with lone electron pairs included in their structure, which could be aromatic. The concept of aromaticity is based on equilibration of planar ring bond lengths due to the presence of shared and delocalized electrons. The number of delocalized electrons could be determined according to Hückel's formula $4 n+2$, where $n=1$ for benzene. There are two commonly used theoretical indexes of aromaticity, the geometric one (HOMA, and closely related parameters, derived from structural dimensions [11-14]) and magnetic [7, 9, 15, 16] (NICS). For benzene, 
HOMA is close to 1. HOMHED is a reparametrized version of HOMA and is dedicated to heterocyclic molecules. Magnetic indexes of aromaticity are usually calculated in the middle of ring as $\operatorname{NICS}(0), 1 \AA$ above that point, $\mathrm{NICS}(1)$, or its zzcomponent, NICS $(1)_{\mathrm{zz}}$. Negative values of NICS are calculated for aromatic compounds while positive for anti-aromatic molecular systems. Another magnetic probe of aromaticity is nuclear shielding and chemical shift of helium-3 atom [17-21]. Studies of aromaticity using ${ }^{3} \mathrm{He}$ are not so popular but they possess one advantage: in principle the predicted ${ }^{3} \mathrm{He}$ NMR chemical shifts could be verified by experiment $[17,18$, 22].

For example, a good agreement was observed between a position of a narrow resonance signal in a ${ }^{3} \mathrm{He}$ NMR spectra of He@C60, as well as for other fullerenes and theoretically predicted isotropic value of helium-3 nuclear magnetic shieldings, converted to chemical shift [20, 21, 23-25]. In many experimental studies, the fullerene cage size, symmetry, and aromaticity in the presence of the encapsulated ${ }^{3} \mathrm{He}$ atom [20, 21, 23, 24] was studied by noble gas NMR technique. In such works, the ${ }^{3} \mathrm{He}$ atom was treated as another, independent magnetic probe of aromaticity.

In Fig. 1 are presented six optimized tautomeric structures of 5-fluorouracil, which is the most important 5-halogeno derivative of uracil. The structures are labeled with the commonly used abbreviations [5]. From the six potential uracil and its 5XU tautomers [26-32], the diketo form (tautomer No 1 in Fig. 1) is the most stable, as verified by experiment and theoretical calculations. On the other hand, it is possible to guess that tautomer No 6 should be the most aromatic one [5]. This

Fig. 1 B3LYPD3/aug-cc-pVQZ optimized tautomers of 5 fluorouracil in the gas phase. The individual tautomers are labeled below tautomer is formed by three alternating single and double bonds, respectively. However, it is difficult "to guess" quantitatively the amount of aromaticity for tautomers 1 and 6 from the formulas, presented in Fig. 1, only. Besides, the nature of $5 \mathrm{X}$ substituent should also alter the aromaticity of the corresponding uracil derivatives. Moreover, the magnitude of magnetic shielding in the vicinity of uracil plane is related to the degree of electron delocalization and it should change perpendicularly to the plane with the distance $R$ from the ring center. These phenomena could be modeled by so-called scanning experiment based on calculation of $\operatorname{NICS}(R)$ value as function of varied distance $R[16,33,34]$ or by the change of ${ }^{3} \mathrm{He}$ isotropic nuclear magnetic shielding $\sigma_{R}$, often converted to chemical shift $\delta_{R}$, when moving the helium probe by a distance $R$ above the ring center [17, 18].

Recently, the aromaticity of acenes was correlated with the HOMO-LUMO energy gap $E_{g}$ [22]. In case of $5 \mathrm{XU}$, by changing the electron-donating properties of $\mathrm{X}$ substituent, the magnitude of $E_{g}$ and electron delocalization within the uracil plane should also vary [5]. Thus, by changing the halogen substituent one could expect a direct impact on $E_{g}$, as well as on the aromaticity of 5-halogenouracils. However, the above mentioned problems were not discussed explicitly in the literature [5] or used to explain some structural and spectroscopic properties of $5 \mathrm{XU}$ molecules.

The aim of the current study is to characterize the aromaticity of uracil and its $5 \mathrm{X}$-derivatives using geometric index of aromaticity HOMHED [35]. Additionally, a profile of magnetic field above the uracil ring will be modeled in separate scan experiments for NICS indexes and helium-3 chemical
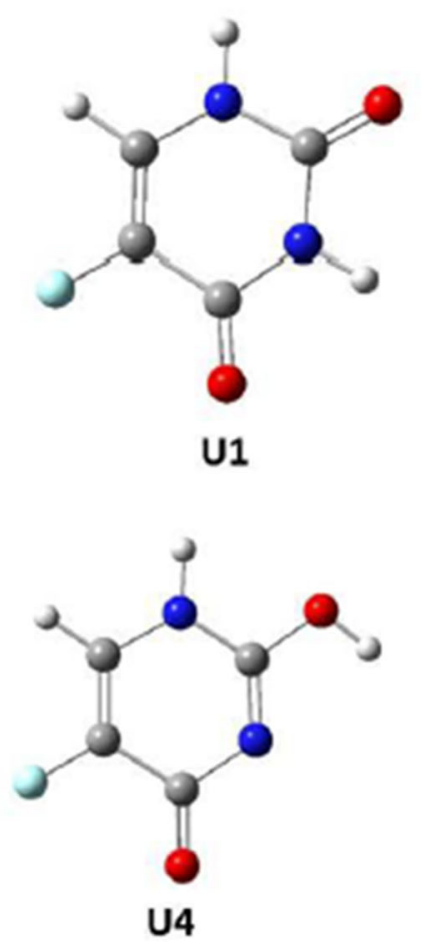
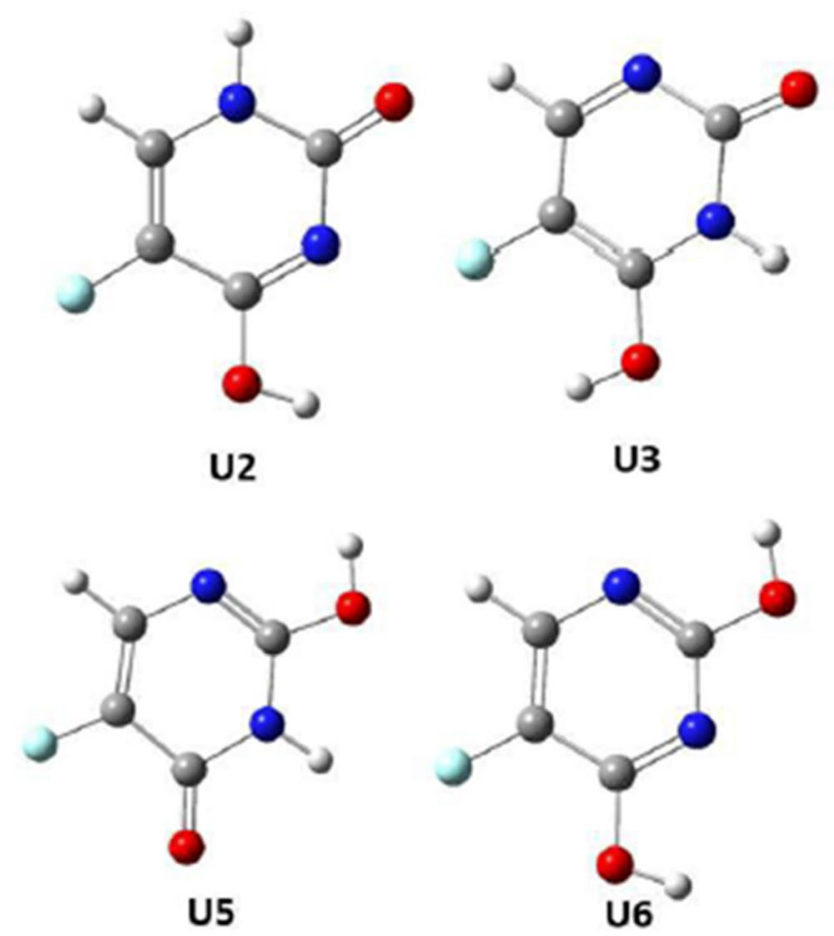
shift. The electron-donating or electron-accepting nature of $\mathrm{X}$ substituent could also influence the magnitude of charge at $\mathrm{C} 5$ atom, as well as ring aromaticity. Thus, the changes of charge at $\mathrm{C} 5$ and $\mathrm{X}$ atoms will be studied using natural bond orbital [36] concept (NBO). Finally, we will look for same correlations between uracil HOMO/LUMO gap, electronegativity of halogen substituent and aromaticity. Since solvent affects aromaticity indexes very little, we will model our compounds in vacuum, and only in some cases compare the results to those, obtained with the presence of water, incorporated by polarized continuum model (PCM) [37].

\section{Computational part}

All calculations were performed with Gaussian 16 program [38]. In this study, we selected a popular and fairly reliable B3LYP [39-41] hybrid density functional improved by inclusion of dispersion interactions via added Grimme's GD3 term [42], for modeling of uracil and its four 5-halogeno derivatives in vacuum and in the condense phase. The latter calculations were conducted using a polar surrounding (water) approximately described by PCM model [37]. The presence of all positive harmonic frequencies confirmed that the calculated structures were the true energy minima. Large Dunning-type correlation-consistent valence basis set of quadruple-zeta quality, augmented with a diffuse function (aug-cc-pVQZ) [43] was selected for better characterization of multiple bonds and lone electron pairs. Due to the problems with SCF convergence and optimization of 5-iodouracil tautomers, calculated with larger basis sets, we tested several combinations of available basis sets. Finally, all tautomers of 5 IU were optimized using 6-31 + G(d) for C, N, O, H, and 6-311G for I. We also managed to optimize the 5 IU1 and 5 IU6 tautomers with two larger basis sets, aug-cc-pVQZ for $\mathrm{C}, \mathrm{H}, \mathrm{N}$, and $\mathrm{O}$, and aug-cc-pVDZ-PP for I. The latter basis set was downloaded from EMSL basis set exchange [44, 45]. Magnetic index of aromaticity NICS [7, 9] was used to determine the aromaticity of the studied tautomers and monohalogenoderivatives of benzene as reference. In addition, geometric indexes of aromaticity, HOMA [12-14] and HOMHED [35], were calculated. NBO charges [36] were calculated at optimized geometries. Gauge including atomic orbital $[46,47]$ (GIAO) NMR calculations of NICS parameters and ${ }^{3} \mathrm{He}$ isotropic nuclear magnetic shieldings at the ring center and above, at distance of $1 \AA$ and $R$, from tautomers 1 and 6 of uracil and 5XU were also performed at the B3LYP/aug-cc-pVQZ level of theory (only for $5 \mathrm{IU}$, a previously mentioned $6-31+\mathrm{G}(\mathrm{d})$ for $\mathrm{C}$, $\mathrm{N}, \mathrm{O}, \mathrm{H}$, and $6-311 \mathrm{G}$ for $I$ basis sets were used). The distance $R$ from the middle of rings was varied from 0 to $2 \AA$ with steps of $0.05 \AA$, from 2 to $5 \AA$ with step of $0.5 \AA$ and from 5 to $10 \AA$ with steps of $1 \AA$. In particular, in agreement with our earlier studies $[17,18]$, the theoretical chemical shifts of ${ }^{3} \mathrm{He}$ in the gas phase $\left(\delta_{\mathrm{He}}\right)$ were obtained using the isotropic value of free probe atom nuclear magnetic shielding $\left(\sigma_{o}\right)$ as reference and calculating its shielding $\sigma_{R}$ at a distance $R$ from the middle of ring, according to the following formula

$\delta_{H e}=\sigma_{o}-\sigma_{R}$.

At that point, we want to stress that the geometry of uracil and 5XU derivatives was optimized at B3LYPD3/aug-ccpVQZ level of theory, but the subsequent GIAO NMR calculations were conducted without Grimme correction term since the results obtained with, and without dispersion corrections, were the same.

For graphical correlations, both linear and nonlinear least square fits were performed and their quality assessed from the corresponding $R^{2}$ parameter. The latter one used second order polynomial formula, abbreviated as polynomial_ 2 .

\section{Results and discussion}

\section{HOMA, HOMHED, and NICS parameters of uracil and $5 X U$}

The calculated HOMHED parameters for the studied structures are gathered in Table 1. It is apparent from Table 1 that all tautomers of uracil and its 5-halogeno derivatives are to some extent aromatic, as reflected by their fairly high HOMHED index (between 0.7 and 1). Following the suggestion of an anonymous reviewer, we also checked the aromaticity of 5FU6 tautomer with $\mathrm{OH}$ group at $\mathrm{C} 4$ position rotated and directed toward the $\mathrm{X}$ atom. This molecule was only negligibly less aromatic than the one with opposite direction of hydroxyl substituent (HOMHED of 0.984 vs. 0.987 for 5FU6). However, comparing the magnitudes of calculated aromaticity indexes, there is a clear difference between the most stable tautomer 1 and 6 . Thus, lower values of

Table 1 B3LYP-D3/aug-cc-pVQZ calculated HOMHED parameters in the gas phase for uracil and all tautomers of $5 \mathrm{XU}$ with benzene included as reference

\begin{tabular}{llllllll}
\hline & 5XU1 & 5XU2 & 5XU3 & 5XU4 & 5XU5 & 5XU6 & 5XU6 $^{\mathbf{a}}$ \\
\hline Uracil & 0.769 & 0.830 & 0.830 & 0.724 & 0.807 & 0.991 & 0.988 \\
$5 \mathrm{FU}$ & 0.758 & 0.822 & 0.829 & 0.736 & 0.801 & 0.987 & 0.984 \\
$5 \mathrm{ClU}$ & 0.755 & 0.825 & 0.883 & 0.712 & 0.802 & 0.998 & 0.984 \\
$5 \mathrm{BrU}$ & 0.756 & 0.827 & 0.832 & 0.711 & 0.801 & 0.989 & 0.984 \\
$5 \mathrm{IU}$ & $0.731^{\mathrm{b}}$ & $0.811^{\mathrm{c}}$ & $0.811^{\mathrm{c}}$ & $0.702^{\mathrm{c}}$ & $0.794^{\mathrm{c}}$ & $0.994^{\mathrm{c}}$ & $0.984^{\mathrm{b}}$ \\
Benzene & 1.000 & & & & & & \\
\hline
\end{tabular}

${ }^{\text {abc }} \mathrm{OH}$ group at $\mathrm{C} 4$ pointing toward $\mathrm{X}$ atom; aug-cc-pVQZ for $\mathrm{C}, \mathrm{N}, \mathrm{O}, \mathrm{H}$ and aug-cc-pVDZ-PP for I; 6-31 + G* basis set for all atoms and 6-311G for I 
HOMEHED (about 0.76 and 0.72 ) are calculated for 5XU1 and 5XU4 tautomers. On the other hand, these indexes of aromaticity are about 0.99 for tautomers 6 and are close to the value calculated for benzene (HOMEHED $\sim 1$ ). The same order of aromaticity for the studied tautomers was earlier reflected by the calculated NICS and HOMA values in ref. [5]

In order to test a potential correlation between geometric parameters of aromaticity and the magnetic ones, in Fig. 2 we plotted the HOMA and HOMHED values for all tautomers of uracil and 5FU vs. the calculated NICS(1) values. The HOMHED parameters were calculated in the current study and both NICS and HOMA ones were taken from our earlier work [5].

Interestingly, it is apparent from Fig. 2 that no good correlation between geometric HOMA, HOMHED, and magnetic index of aromaticity NICS(1) exists. However, the corresponding $R^{2}$ parameter for a dependence between $\operatorname{NICS}(1)$ calculated for all studied 5XU tautomers and geometric indexes is higher for HOMHED than for HOMA (0.82 vs. 0.67). Probably, this also illustrates the multidimensional character of aromaticity derived by using various parameters and points out to some advantage of using HOMHED combined with NICS(1) for characterization of heterocyclic compounds instead of traditional HOMA descriptor.

\section{NICS scan above molecular planes of uracil and 5XU}

A more clear picture of aromaticity profile above $5 \mathrm{FU}$ ring is shown in Fig. 3A and it somehow represents a "magnitude" of ring current at different distances from the molecular plane, e.g., above the center of the ring. As result of "scan experiment $[16-18,33,34]$ " it is possible to show a characteristic profile of $\operatorname{NICS}(R)$ dependence on the distance $R$ above the ring center of tautomers 1 and 6 of uracil, as well as benzene, taken as a model compound with a NICS showing a minimum

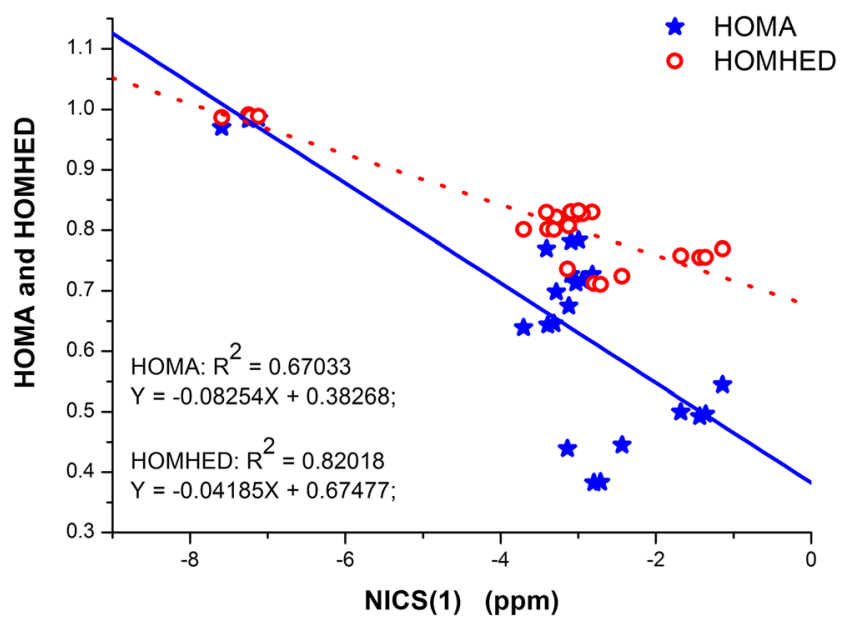

Fig. 2 Dependence of HOMA and HOMHED vs. NICS(1) for 5XU tautomers together with the determined parameters and fitting lines of linear correlations shown
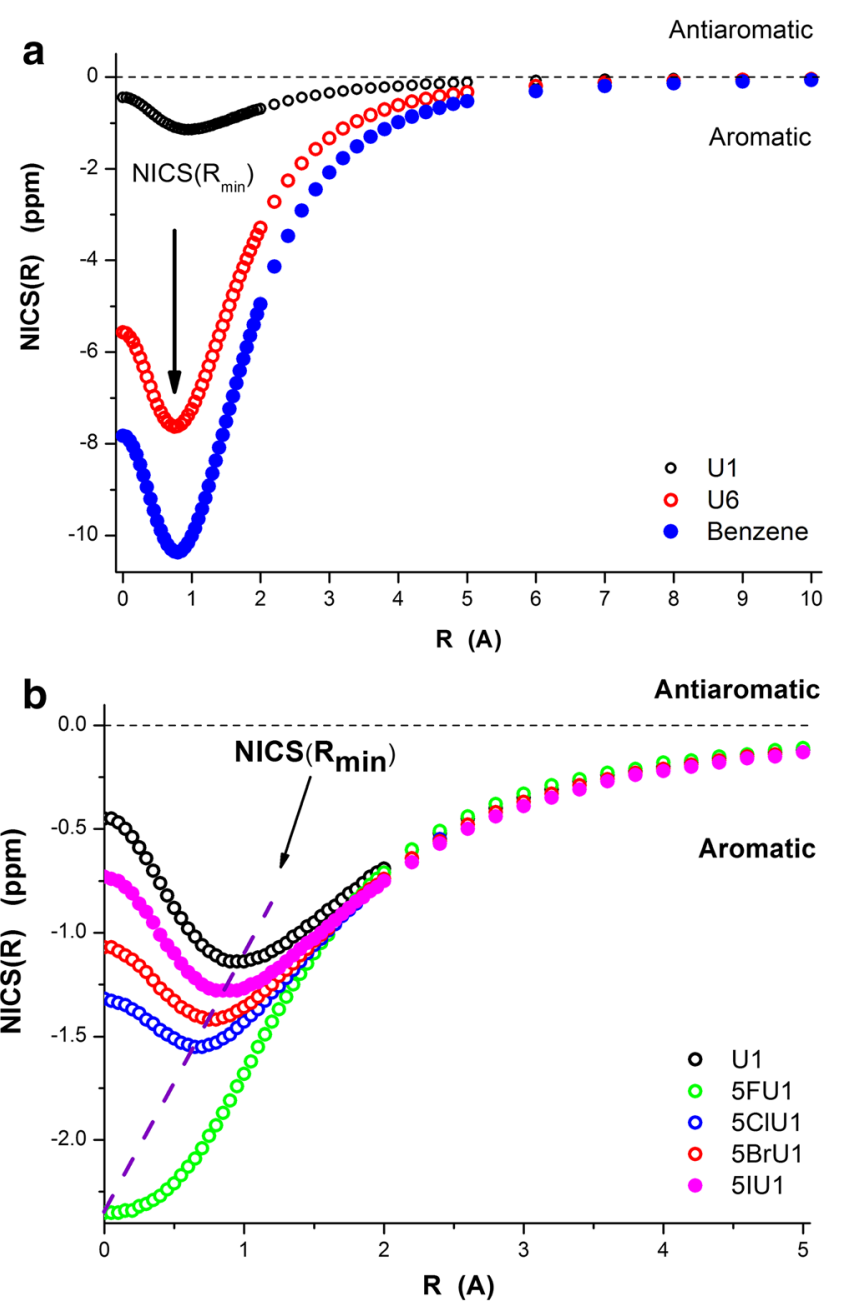

Fig. 3 Dependence of B3LYP-D3/aug-ccpVQZ calculated NICS $(R)$ in vacuum on the distance $R$ from the center of the uracil ring for: $\mathbf{A}$ uracil tautomers 1, 6 and benzene; $\mathbf{B}$ tautomer 1 of uracil and the remaining $5 \mathrm{XU} 1$ derivatives (the arbitrary placed red dashed line goes through the curve minima)

at $R_{\min }$ distance, markedly below $1 \AA$. The "scan" curve resembles a "Morse curve" and is related to the aromaticity profile above (and below) the benzene ring [10, 16-18, 34]. However, in case of the diketo tautomer of uracil (U1), the minimum is very shallow what indicates a very small aromaticity. Interestingly, on the contrary to tautomer 1, tautomer 6 shows a scan curve closely resembling benzene. Thus, it is apparent that the significant depth of scan minimum, $\operatorname{NICS}\left(R_{\min }\right)$ of about -7.5 ppm for tautomer 6 indicates its high aromaticity while tautomer $\mathbf{1}$ is significantly less aromatic $\left(\operatorname{NICS}\left(R_{\min }\right)\right.$ is about $\left.-1 \mathrm{ppm}\right)$. Evidently, the curve depth of about $-11 \mathrm{ppm}$ and pattern shape depends on the $\pi$ electron current density, being larger in benzene (NICS $\left(R_{\min }\right)$ than in tautomer 6 of uracil. In Fig. 3B, the results of similar "scan" experiments for tautomer $\mathbf{1}$ of all the studied 5XU1 compounds are shown. Analogous $\operatorname{NICS}(R)$ curve shapes with minimum in the range of $R=0.5$ and $1 \AA$ are observed for all 5XU except the tautomer 1 of 5 - 
fluorouracil (Fig. 3B). In the latter case the minimum is observed at $R=0 \AA$. The same situation was observed for pyrrole [18]. Besides, in that study we demonstrated that the position $\left(R_{\mathrm{min}}\right)$ and magnitude of $\operatorname{NICS}\left(R_{\mathrm{min}}\right)$ could be roughly correlated with the aromaticity degree of ring compounds [18]. Analyzing curve patterns from Fig. 3B, one could observe some systematic changes of $R_{\min }$ and $\operatorname{NICS}\left(R_{\min }\right)$ parameters with the type of $\mathrm{X}$ substituent. Thus, the dashed red line, placed in the figure for the reader's convenience, starts at $R_{\min }=0 \AA$ and goes nicely through all the curves minima.

At this point, we also tested the impact of water, modeled by PCM, on the aromaticity of uracil's tautomer 1 and 6 . The scan experiment for $R$ changing from 0 to $2 \AA$ was performed in vacuum and in water (see Fig. S1A and B in the supplementary material). The NICS $(R)$ scan curves calculated in the presence of water nearly coincide with those, obtained in the gas phase. Thus, the subsequent calculations were performed in the gas phase only since the used model indicates a lack of effect of polar solvent on the aromaticity of uracil.

One could also expect a direct correlation between electronegativity of halogen substituent and the density of $\pi$-electron ring current, as reflected in "NICS scan experiments". In fact, nice linear $\left(R^{2}>0.9\right)$ dependences of $R_{\min }$ and $\operatorname{NICS}\left(R_{\min }\right)$ vs. Pauling's electronegativity of $\mathrm{X}$ substituent are observed in Fig. 4A and B. The increase of X substituent electronegativity results in decreasing $R_{\min }$ and a small increase of ring aromaticity (see Fig. 4B), as reflected by more negative value of $\operatorname{NICS}\left(R_{\min }\right)$.

To support our data for $5 \mathrm{XU}$ molecular systems we also checked the impact of halogen electronegativity on monohalogenobenzenes $\mathrm{C}_{6} \mathrm{H}_{5} \mathrm{X}$, where $\mathrm{X}=\mathrm{H}, \mathrm{I}, \mathrm{Br}, \mathrm{Cl}$, and $\mathrm{F}$. The NICS $(R)$ scan experiment produced similar curve profiles to those, presented in Fig. 3B (see Fig. S2 in the Supplementary Material). However, the minima for halogenobenzenes were significantly lower (more negative) than for 5XU1 indicating a higher degree of aromaticity for these compounds (NICS $\left(R_{\min }\right)$ from about -9 to $-11 \mathrm{ppm}$ ). In addition, for $\mathrm{C}_{6} \mathrm{H}_{5} \mathrm{~F}$, the calculated $R_{\min } \sim 0.7 \AA$. Besides, strong linear correlations between $R_{\min }, \operatorname{NICS}\left(R_{\min }\right)$ and electronegativity were observed for monohalogenobenzenes (Fig. $\mathrm{S} 3$ in the supplementary material).

\section{Electronegativity of $X$ substituent and NBO charge at C5 atom}

It is also important to check the impact of X substituent electronegativity on NBO charge at $\mathrm{C} 5$ atom in the most stable tautomer of $5 \mathrm{XU} 1$ molecules. It is apparent from Fig. 5 that changes of NBO charge of X substituent is reflected in formation of almost equal but opposite sign charge at carbon No 5 . The observed changes of charge are very regular and point to the electronegativity as the main factor controlling the electronic impact on the halogeno-substituted uracil ring. The
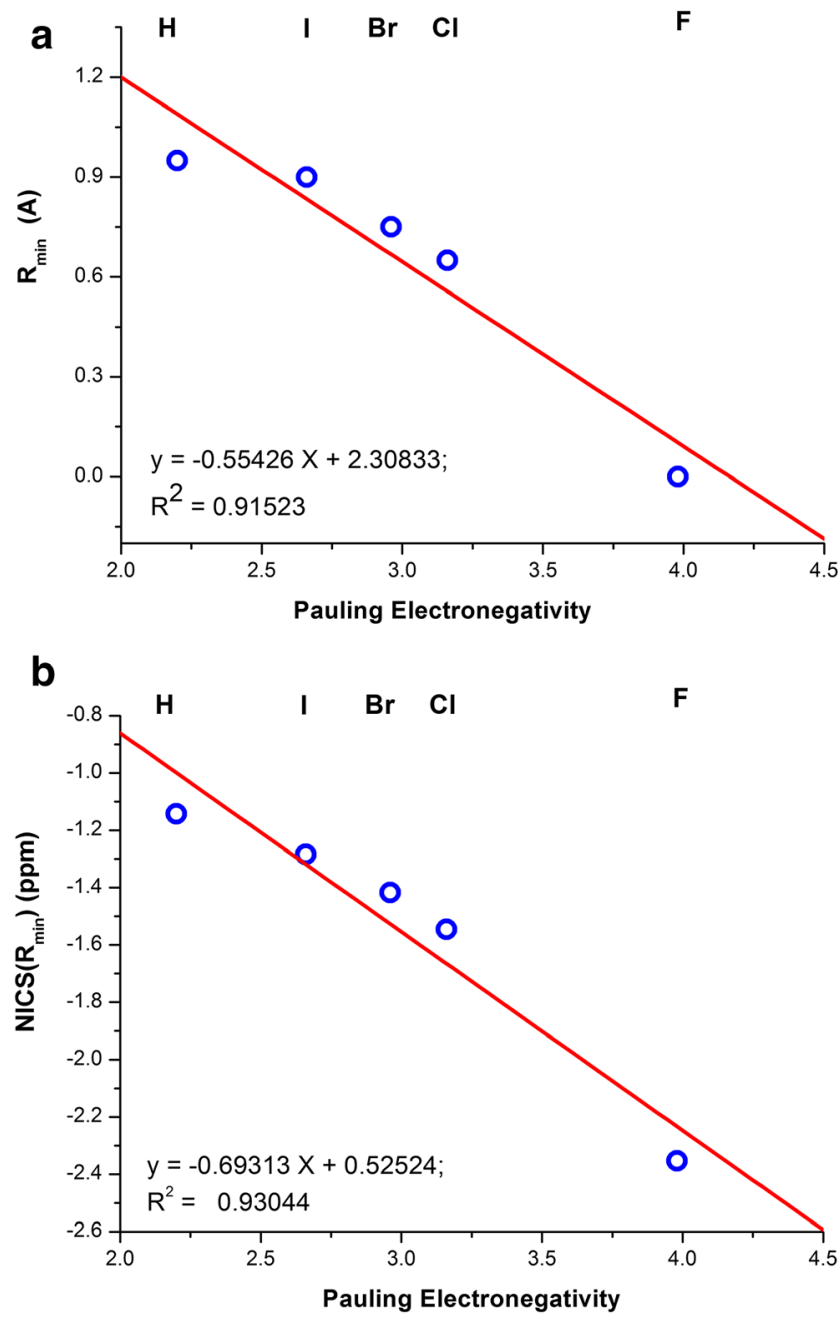

Fig. 4 Linear correlation between Pauling electronegativity of $\mathrm{X}$ substituent and $\mathbf{A} R_{\min }$ and $\mathbf{B} \operatorname{NICS}\left(R_{\min }\right)$ for tautomers 1 of $5 \mathrm{XU}$

difference of charges $(\mathrm{X}-\mathrm{C} 5)$ also decreases in a regular, nonlinear way. Here, we want to stress, that in this case, no

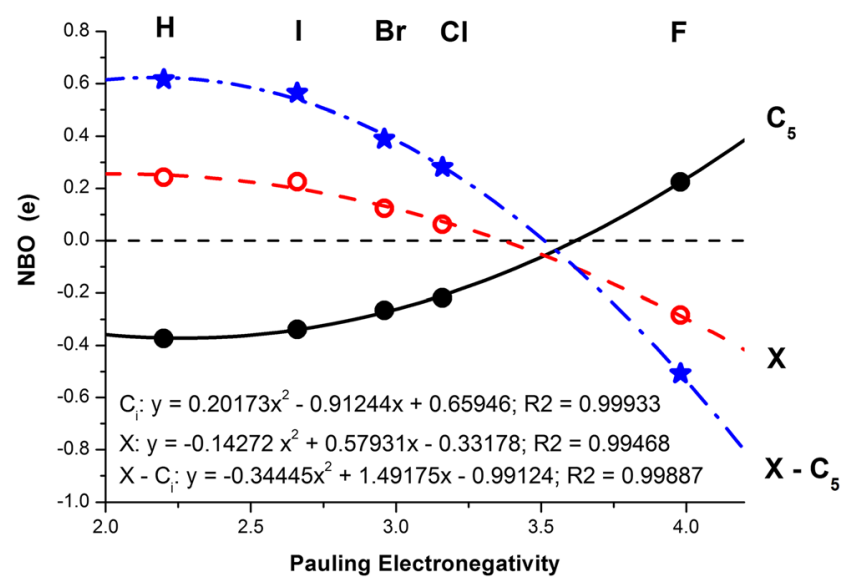

Fig. 5 Nonlinear dependence between NBO charge of X substituent, C5 atom, their difference $(\mathrm{X}-\mathrm{C} 5)$, and electronegativity of $\mathrm{X}$ substituent in tautomer 1 of $5 \mathrm{XU}$ 
linear dependence exist. Instead, a second-order polynomial nicely fits the calculated points $\left(R^{2}>0.99\right)$.

The observed patterns of NBO charge variations at $\mathrm{C} 5$ are similar to those for benzene ring carbon atom, directly bonded to halogen (see Fig. S4 in the supplementary material).

Our results are also in agreement with earlier studies on electronic effect of $\mathrm{X}$ substituent, in particular its donating and accepting properties, changing only to a little extend the aromaticity of benzene ring [48-51]. On the other hand, olefins are significantly more sensitive to such effects, including "push-pull cases") [52].

Therefore, the above data strongly point out to halogen electronegativity (and fluorine as an extreme case), reflected as an inductive effect (I), being responsible for small changes of aromaticity in 5-halogenouracils. On the other hand, halogen atoms also produce a resonance (mezomeric) effect $(R)$ which is absent for $\mathrm{X}=\mathrm{H}$. The order of this effect is $\mathrm{F}>\mathrm{Cl}>$ $\mathrm{Br}>\mathrm{I}$. Thus, each substituent shows a competing $I$ and $R$ effects and fluorine is an extreme example. It is also worth mentioning that in case of borazine derivatives, the fluorine substituent also resulted in $\operatorname{NICS}(R)$ scan curve with a minimum at $R=0 \AA$ [53] (the reported curve patterns were similar to that in Fig. 3B). The behavior of F-substituent somehow differs from the other halogens probably because of its extremely low electronegativity. However, the fluorine anomaly is well known in the literature (see its anomalous reactivity in ref. [54]).

\section{${ }^{3} \mathrm{He}$ atom as a magnetic probe of uracil and $5 \mathrm{XU}$ aromaticity}

Despite general acceptance of aromaticity as a very important description of structural, energetic and spectroscopic properties of chemical compounds and their reactivity, there exists many controversies in this field $[10,51]$. Aromaticity is often regarded as a multidimensional property. Thus, sometimes no direct correlation between the individual indexes of aromaticity could be seen. In this regard, in the current study we were interested to see, if there is some correlation between the geometric indexes of aromaticity for uracil and its 5-halogeno derivatives and their two, apparently not related magnetic indexes-NICS and helium-3 nuclear shieldings.

At this stage of our study, the helium-3 atom has been used as another magnetic probe of aromaticity. Isotropic values of ${ }^{3} \mathrm{He}$ nuclear magnetic shielding constants for the helium atom located $1 \AA$ above the ring center for uracil and its tautomers, as well as for the remaining $5 \mathrm{XU}$ molecules were calculated. For brevity, in Table $\mathrm{S} 1$ in the supporting material are shown ${ }^{3} \mathrm{He}$ isotropic nuclear magnetic shieldings and chemical shifts of helium-3 atom $1 \AA$ above the ring center (calculated with respect to isolated probe in the gas phase). In order to test a correlation between two magnetic indexes of aromaticity, $\delta\left({ }^{3} \mathrm{He}\right)$ and the NICS(1) for all tautomers of uracil and $5 \mathrm{XU}$, in Fig. 6 were plotted the calculated parameters and fitted using a linear least square (LSQ) procedure. As result, a very good linear correlation between the two magnetic parameters of aromaticity, calculated in vacuum was produced $\left(R^{2}=\right.$ $0.98888)$. This result suggests that in the future carefully designed experiments, ${ }^{3} \mathrm{He}$ NMR spectroscopy could be used to determine experimentally the "magnitude of aromaticity" of the studied molecular systems. In other words, we postulate to use helium-3 not only as a computational but also an experimental magnetic probe of aromaticity.

On the contrary, a significantly worse linear correlation between the chemical shift of the ${ }^{3} \mathrm{He}$ atom $1 \AA$ above the middle of ring and the geometric index of aromaticity, HOMHED is apparent from Fig. 7A $\left(R^{2}=0.84\right)$. This correlation is even worse for HOMA (not shown, $R^{2}=0.71$ ).

\section{HOMO-LUMO energy gap and aromaticity of uracil and 5XU1}

Recently, we reported on exponential correlation between $E_{g}$ and NICS index calculated for a series of acenes [22]. In the current studies, we also expected a similar correlation between $E_{g}$ and aromaticity indexes of uracil tautomers and their derivatives. In Table S2 are gathered B3LYP-D3/aug-cc-pVQZ calculated HOMO, LUMO, and $E_{g}$ energies for uracil tautomers, as well as their derivatives in the gas phase, and in Fig. 7B, a dependence between NICS(1) and $E_{g}$ is plotted. The results presented in Fig. 7B are very scattered and once more demonstrate the fact that aromaticity is a very complicated and multidimensional phenomenon [10]. Contrary to the earlier work on regularly growing size of conjugated acenes [22], no correlation could be observed between $E_{g}$ and the calculated indexes of aromaticity for all tautomers of uracil

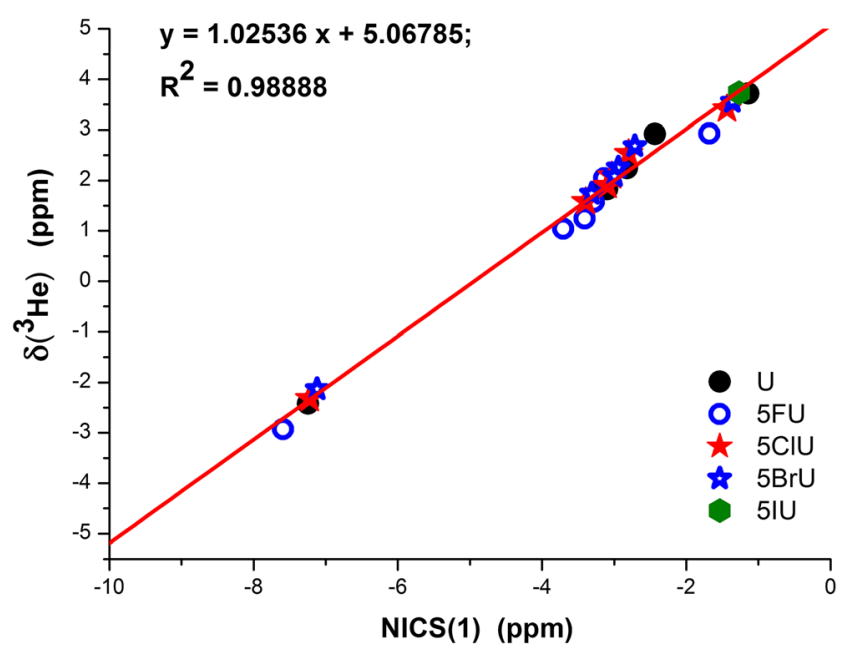

Fig. 6 Linear dependence between B3LYP/aug-cc-pVQZ calculated $\delta\left({ }^{3} \mathrm{He}\right)$ for helium atom located at a distance of $1 \AA$ above the middle of $5 \mathrm{XU}$ heterocyclic ring and NICS(1) 

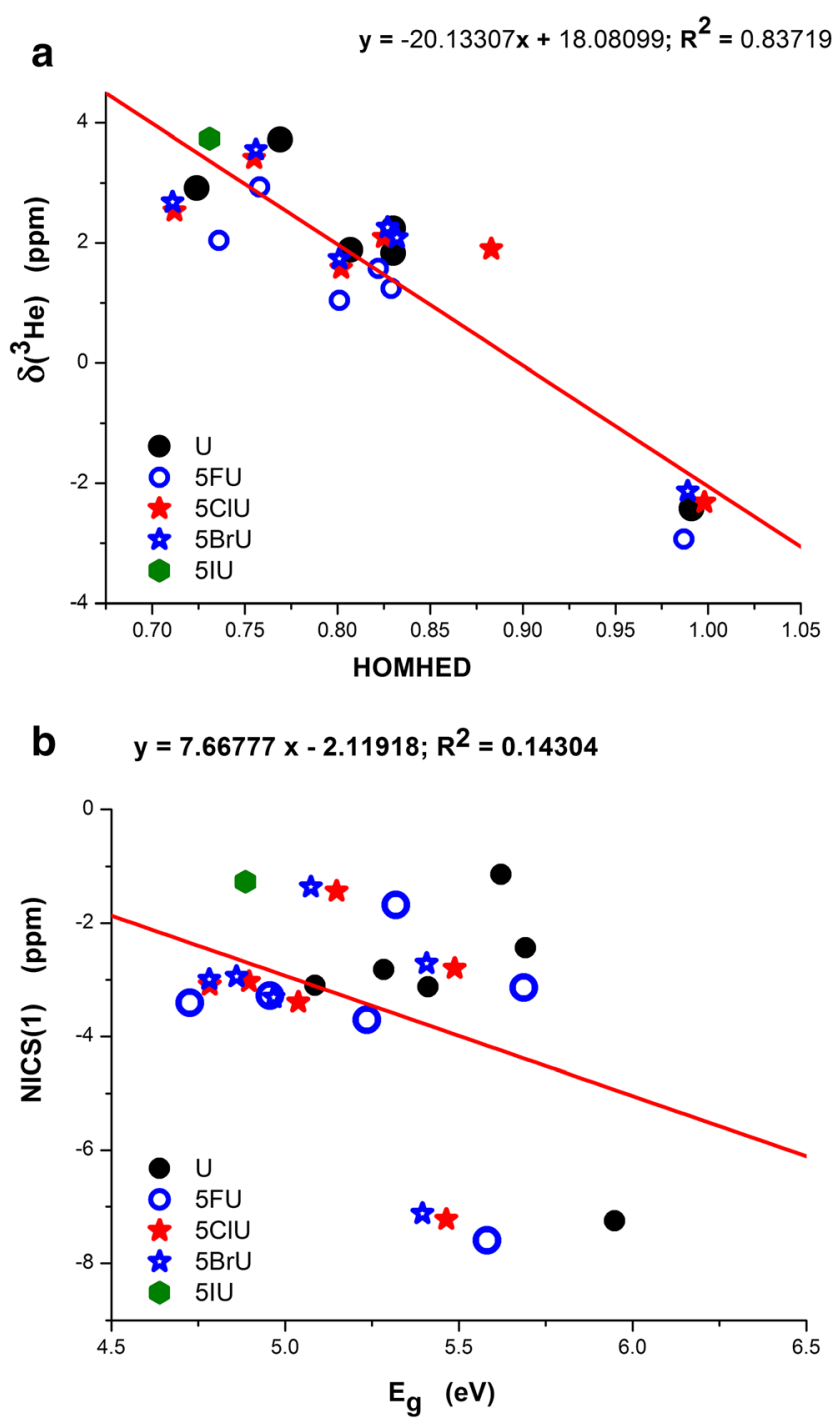

Fig. 7 A A weak linear correlation between two aromaticity probes for the studied 5XU tautomers, $\delta\left({ }^{3} \mathrm{He}\right)$ calculated $1 \AA$ about ring center and HOMHED, and B NICS(1) vs. $E_{g}$

and 5-halogenuracils (an attempt of the linear fit produced RMS of only 0.14304$)$. However, by analyzing $E_{g}$ and NICS(1) of tautomer 1 only a nice linear correlation is observed with $R^{2}$ of 0.92 (See Fig. S5 in the supplementary material).

\section{Conclusions}

DFT modeling of molecular structure of six tautomers of uracil and its 5-halogeno derivatives was performed at DFT (B3LYPD3/aug-cc-pVQZ) level of theory. The aromaticity of the studied heterocyclic compounds was determined using geometric indexes of aromaticity, HOMA, and HOMHED. The most stable diketo tautomer 5XU1 showed very small aromaticity (HOMA and HOMHED about 0.5 and 0.76 , respectively) while the "rare" U6 one was fairly aromatic (HOMA and HOMHED close to 1). GIAO NMR calculations of magnetic indexes of aromaticity, $\operatorname{NICS}(0), \operatorname{NICS}(1), \operatorname{NICS}(1) z z$, and $\operatorname{NICS}(R)$, for $R=0$ to $10 \AA$ with step of $0.05 \AA$ were also conducted on the previously optimized structures at B3LYP/aug-cc-pVQZ level of theory, e.g., without dispersion effects included. Electronegativity of $\mathrm{X}$ substituent and its induction effect was the main factor controlling the charge at $\mathrm{C} 5$ atom in $5 \mathrm{XU}$ tautomers. Regular changes of NBO charges at $\mathrm{C} 5$ (nicely fitted with second order polynomial formula), with respect to electronegativity of $\mathrm{X}$ substituent were observed for tautomer 1 of $5 \mathrm{XU}$ structures.

The last magnetic probe of aromaticity used was isotope of helium-3. Its nuclear shielding at $R=1 \AA$, as well as scans up to $10 \AA$ were also calculated and converted to helium chemical shifts.

Both the geometric indexes of aromaticity and the magnetic ones reflected low aromaticity of tautomer 1 and high, comparable to benzene for tautomer 5 XU6. High aromaticity of tautomer 6 confirms the suitability of its traditional formula, presented with three alternated single and double bonds. A linear increase of 5XU aromaticity, expressed by $R_{\min }$ and $\operatorname{NICS}\left(R_{\min }\right)$, obtained from scan experiments, with the electronegativity of halogen substituent was observed $\left(R^{2}\right.$ about 0.92 and 0.93$)$.

Linear correlations were observed for $\delta\left({ }^{3} \mathrm{He}\right)$ and $\operatorname{NICS}(1)$ with $R^{2}$ close to $1\left(R^{2}=0.99\right)$. However, the linear correlation between $\delta\left({ }^{3} \mathrm{He}\right)$ and HOMHED with $R^{2}$ of 0.83 was less efficient (correlation for HOMA was even worse, with $R^{2}=0.71$ ). On the contrary, no direct correlation between NICS(1) for all tautomers and $E_{g}$ was observed ( $R^{2}$ about 0.14$)$. On the contrary, a strong linear correlation between these parameters was observed for tautomer 1 .

It was also observed that the presence of a polar environment (water modeled by PCM) increased only negligible the aromaticity of $5 \mathrm{XU}$.

Supplementary Information The online version contains supplementary material available at https://doi.org/10.1007/s11224-020-01682-x.

Acknowledgments Calculations have been carried out using resources provided by the Wrocław Centre for Networking and Supercomputing (http://wcss.pl).

Code availability Gaussian 16 at WCSS Wroclaw.

Authors' contributions K. R.- conducting research and partial analysis of results; A. B.-conducting research and partial analysis of results; $\mathrm{T}$. K.- planning research, analysis results, and preparing a manuscript; $M$. A. B.-planning research, analysis results, and preparing a manuscript.

Data availability From corresponding authors upon request. 


\section{Compliance with ethical standards}

Not applicable. The ethical standards have been met.

Conflict of interest The authors declare that they have no competing interests.

Open Access This article is licensed under a Creative Commons Attribution 4.0 International License, which permits use, sharing, adaptation, distribution and reproduction in any medium or format, as long as you give appropriate credit to the original author(s) and the source, provide a link to the Creative Commons licence, and indicate if changes were made. The images or other third party material in this article are included in the article's Creative Commons licence, unless indicated otherwise in a credit line to the material. If material is not included in the article's Creative Commons licence and your intended use is not permitted by statutory regulation or exceeds the permitted use, you will need to obtain permission directly from the copyright holder. To view a copy of this licence, visit http://creativecommons.org/licenses/by/4.0/.

\section{References}

1. Krokan HE, Drabløs F, Slupphaug G (2002) Uracil in DNA occurrence, consequences and repair. Oncogene 21(58):89358948. https://doi.org/10.1038/sj.onc. 1205996

2. Martins Z, Botta O, Fogel ML, Sephton MA, Glavin DP, Watson JS, Dworkin JP, Schwartz AW, Ehrenfreund P (2008) Extraterrestrial nucleobases in the Murchison meteorite. Earth Planet Sci Lett 270(1):130-136. https://doi.org/10.1016/j.epsl. 2008.03.026

3. Duschinsky R, Pleven E, Heidelberger C (1957) The synthesis of 5fluoropyrimidines. J Am Chem Soc 79(16):4559-4560. https://doi. org/10.1021/ja01573a087

4. Heidelberger C, Chaudhuri NK, Danneberg P, Mooren D, Griesbach L, Duschinsky R, Schnitzer RJ, Pleven E, Scheiner J (1957) Fluorinated pyrimidines, a new class of tumour-inhibitory compounds. Nature 179(4561):663-666

5. Rzepiela K, Buczek A, Kupka T, Broda MA (2020) Factors governing the chemical stability and NMR parameters of uracil tautomers and its 5-halogen derivatives. Molecules 25:3931

6. McMurry J (2007) Organic chemistry7th edn Brooks-Cole.

7. Chen Z, Wannere CS, Corminboeuf C, Puchta R, Schleyer PR (2005) Nucleus-independent chemical shifts (NICS) as an aromaticity criterion. Chem Rev 105(10):3842-3888. https://doi.org/10. 1021/cr030088+

8. Cyrański MK, Krygowski TM, Wisiorowski M, van Eikema Hommes NJR, Von Rague SP (1998) Global and local aromaticity in porphyrins: an analysis based on molecular geometries and nucleus-independent chemical shifts. Angew Chem Int Ed 37: $177-180$

9. Schleyer PvR, Maerker C, Dransfeld A, Jiao H, Hommes NJRvE (1996) Nucleus-independent chemical shifts: a simple and efficient aromaticity probe. J Am Chem Soc 118:6317-6318. doi:https://doi. org/10.1021/ja $960582 \mathrm{~d}$

10. Solà M (2017) Why aromaticity is a suspicious concept? Why? Front Chem 5 (MAR). https://doi.org/10.3389/fchem.2017.00022

11. Raczyńska ED (2019) Application of the extended HOMED (harmonic oscillator model of aromaticity) index to simple and tautomeric five-membered heteroaromatic cycles with $\mathrm{C}, \mathrm{N}, \mathrm{O}, \mathrm{P}$, and $\mathrm{S}$ atoms. Symmetry 11 (2). doi:https://doi.org/10.3390/sym11020146
12. Dobrowolski JC (2019) Three queries about the HOMA index. ACS Omega 4(20):18699-18710. https://doi.org/10.1021/ acsomega.9b02628

13. Kruszewski J, Krygowski TM (1972) Definition of aromaticity basing on the harmonic oscillator model. Tetrahedron Lett 13: 3839-3842

14. Frizzo C, Martins M (2011) Aromaticity in heterocycles: new HOMA index parametrization. Struct Chem 23. https://doi.org/10. 1007/s11224-011-9883-Z

15. Fallah-Bagher-Shaidaei H, Wannere CS, Corminboeuf C, Puchta R, Schleyer PVR (2006) Which NICS aromaticity index for planar $\pi$ rings is best? Org Lett 8(5):863-866. https://doi.org/10.1021/ o10529546

16. Stanger A (2006) Nucleus-independent chemical shifts (NICS): distance dependence and revised criteria for aromaticity and antiaromaticity. J Organomet Chem 71(3):883-893. https://doi. org/10.1021/jo051746o

17. Gajda $Ł$, Kupka T, Broda MA, Leszczyńska M, Ejsmont K (2018) Method and basis set dependence of the NICS indexes of aromaticity for benzene. Magn Reson Chem 56:265-275

18. Kupka T, Gajda $Ł$, Stobiński L, Kołodziej $Ł$, Mnich A, Buczek A, Broda MA (2019) Local aromaticity mapping in the vicinity of planar and nonplanar molecules. Magn Reson Chem 57:359-372

19. Radula-Janik K, Kupka T (2015) ${ }^{3} \mathrm{He}$ NMR studies on heliumpyrrole, helium-indole, and helium-carbazole systems: a new tool for following chemistry of heterocyclic compounds. Magn Reson Chem 53:103-109

20. Saunders M, Jiménez-Vázquez HA, Cross RJ, Mroczkowski S, Freedberg DI, Anet FAL (1994) Probing the interior of fullerenes by $3 \mathrm{He}$ NMR spectroscopy of endohedral 3He@C60 and 3He@C70. Nature 367(6460):256-258. https://doi.org/10.1038/ $367256 \mathrm{a} 0$

21. Sternfeld T, Saunders M, Cross RJ, Rabinovitz M (2003) The inside story of fullerene anions: a $3 \mathrm{He}$ NMR aromaticity probe. Angew Chem Int Ed 42:3136-3139

22. Gajda M, Gajda $Ł$, Kupka T, Kar T (2020) Local aromaticity in polyacenes manifested by individual proton and carbon shieldings: DFT mapping of aromaticity. Magn Reson Chem 51:463-468. https://doi.org/10.1002/mrc.4967

23. Saunders M, Cross RJ, Jiménez-Vázquez HA, Shimshi R, Khong A (1996) Noble gas atoms inside fullerenes. Science 271:1693-1697

24. Saunders M, Jimenez-Vazquez HA, Bangerter BW, Cross RJ, Mroczkowski S, Freedberg DI, Anet FAL (1994) 3He NMR, a powerful new tool for following fullerene chemistry. J Am Chem Soc 116:3621-3622

25. Kupka T, Stachów M, Stobiński L, Kaminský J (2013) 3He NMR: from free gas to its encapsulation in fullerene. Magnetic Resonance in Chemistry : MRC 51:463-468

26. Hanus M, Kabeláč M, Nachtigallová D, Hobza P (2005) Mutagenic properties of 5-halogenuracils: correlated quantum chemical ab initio study. Biochemistry 44(5):1701-1707. https://doi.org/10. 1021/bi048112g

27. Colasurdo DD, Pila MN, Iglesias DA, Laurella SL, Ruiz DL (2017) Tautomerism of uracil and related compounds: a mass spectrometry study. Eur J Mass Spectrom 24(2):214-224. https://doi.org/10. $1177 / 1469066717712461$

28. Leszczynski J (1992) Tautomerism of uracil: the final chapter? Fourth-order electron correlation contributions to the relative energies of tautomers. J Phys Chem 96(4):1649-1653. https://doi.org/ 10.1021/j100183a029

29. Markova N, Enchev V, Timtcheva I (2005) Oxo-hydroxy tautomerism of 5-fluorouracil: water-assisted proton transfer. J Phys Chem A 109(9):1981-1988. https://doi.org/10.1021/jp046132m

30. Muñoz Freán S, Alcolea Palafox M, Rastogi VK (2013) Effect of the microhydration on the tautomerism in the anticarcinogenic drug 5-fluorouracil and relationships with other 5-haloderivatives. J Mol 
Struct 1054-1055:32-45. https://doi.org/10.1016/j.molstruc.2013. 09.008

31. Rastogi VK, Palafox MA (2011) Vibrational spectra, tautomerism and thermodynamics of anticarcinogenic drug: 5-fluorouracil. Spectrochim Acta Part A: Mol Biomol Spectrosc 79(5):970-977. https://doi.org/10.1016/j.saa.2011.04.008

32. Yekeler H, Özbakır D (2001) Concerning the solvent effect in the tautomerism of uracil, 5-fluorouracil, and thymine by densityfunctional theory and ab initio calculations. J Mol Model 7(4): 103-111. https://doi.org/10.1007/s008940100015

33. Acke G, Van Damme S, Havenith RWA, Bultinck P (2019) Quantifying the conceptual problems associated with the isotropic NICS through analyses of its underlying density. Phys Chem Chem Phys 21(6):3145-3153. https://doi.org/10.1039/c8cp07343k

34. Gershoni-Poranne R, Stanger A (2015) Magnetic criteria of aromaticity. Chem Soc Rev 44(18):6597-6615. https://doi.org/10.1039/ c5 $\operatorname{cs} 00114 \mathrm{e}$

35. Frizzo CP, Martins MAP (2012) Aromaticity in heterocycles: new HOMA index parametrization. Struct Chem 23(2):375-380. https:// doi.org/10.1007/s11224-011-9883-Z

36. Weinhold F, Landis CR (2001) Natural bond orbitals and extensions of localized bonding concepts. Chem Educ Res Pract 2(2):91104. https://doi.org/10.1039/B1RP90011K

37. Tomasi J, Mennucci B, Cammi R (2005) Quantum mechanical continuum solvation models. Chem Rev 105(8):2999-3094. https://doi.org/10.1021/cr9904009

38. Frisch MJ, Trucks GW, Schlegel HB, Scuseria GE, Robb MA, Cheeseman JR, Scalmani G, Barone V, Petersson GA, Nakatsuji H, Li X, Caricato M, Marenich AV, Bloino J, Janesko BG, Gomperts R, Mennucci B, Hratchian HP, Ortiz JV, Izmaylov AF, Sonnenberg JL, Williams, Ding F, Lipparini F, Egidi F, Goings J, Peng B, Petrone A, Henderson T, Ranasinghe D, Zakrzewski VG, Gao J, Rega N, Zheng G, Liang W, Hada M, Ehara M, Toyota K, Fukuda R, Hasegawa J, Ishida M, Nakajima T, Honda Y, Kitao O, Nakai H, Vreven T, Throssell K, Montgomery Jr. JA, Peralta JE, Ogliaro F, Bearpark MJ, Heyd JJ, Brothers EN, Kudin KN, Staroverov VN, Keith TA, Kobayashi R, Normand J, Raghavachari K, Rendell AP, Burant JC, Iyengar SS, Tomasi J, Cossi M, Millam JM, Klene M, Adamo C, Cammi R, Ochterski JW, Martin RL, Morokuma K, Farkas O, Foresman JB, Fox DJ (2016) Gaussian 16 Rev. C.01. Gaussian 16 Rev. C.01. Wallingford, CT

39. Becke AD (1988) Density-functional exchange-energy approximation with correct asymptotic behavior. Phys Rev A 38:3098-3100

40. Lee C, Yang, W., and Parr, R. G. (1988) Development of the ColleSalvetti correlation-energy formula into a functional of the electron density. Phys Rev B 37 (2):785-789

41. Miehlich B, Savin A, Stoll H, Preuss H (1989) Results obtained with the correlation-energy density functionals of Becke and Lee, Yang and Parr. Chem Phys Lett 157:200-206
42. Grimme S, Antony J, Ehrlich S, Krieg S (2010) A consistent and accurate $a b$ initio parametrization of density functional dispersion correction (dft-d) for the 94 elements H-Pu. J Chem Phys 132: 154104

43. Kendall RA, Dunning Jr TH, Harrison RJ (1992) Electron affinities of the first-row atoms revisited. Systematic basis sets and wave functions. J Chem Phys 96:6796-6806

44. EMSL. EMSL basis set exchange. https://bse.pnl.gov.bse/portal. Accessed many times in 2019 and 2020

45. Schuchardt KL, Didier BT, Elsethagen T, Sun L, Gurumoorthi V, Chase J, Li J, Windus TL (2007) Basis set exchange: a community database for computational sciences. J Chem Inf Model 47:10451052

46. Ditchfield R (1974) Self-consistent perturbation theory of diamagnetism I. A gauge-invariant LCAO method for N.M.R. chemical shifts. Mol Phys 27:789-807

47. Wolinski K, Hinton JF, Pulay P (1990) Efficient implementation of the gauge-independent atomic orbital method for NMR chemical shift calculations. J Am Chem Soc 112(23):8251-8260

48. Manassir M, Pakiari AH (2019) An electronic properties investigation to interpret the substituent constants of monosubstituted benzene derivatives. J Mol Graphic Model 92:201-207. https://doi.org/ 10.1016/j.jmgm.2019.07.017

49. Krygowski TM, Ejsmont K, Stepień BT, Cyrański MK, Poater J, Solà M (2004) Relation between the substituent effect and aromaticity. J Organomet Chem 69:6634-6640

50. Zarate X, MacLeod-Carey D, Muñoz-Castro A, Schott E (2019) Understanding the aromaticity of C6X6 (X = H, F, Cl, Br, I). Insights from different theoretical criteria. Chem Phys Lett 720: 52-57. https://doi.org/10.1016/j.cplett.2019.01.058

51. Shishkin OV, Omelchenko IV, Krasovska MV, Zubatyuk RI, Gorb L, Leszczynski J (2006) Aromaticity of monosubstituted derivatives of benzene. The application of out-of-plane ring deformation energy for a quantitative description of aromaticity. J Mol Struct 791(1):158-164. https://doi.org/10.1016/j.molstruc.2006.01.019

52. Siodla T, Szatylowicz H, Varaksin KS, Krygowski TM (2016) Difference in pi-electron delocalization for monosubstituted olefinic and aromatic systems. RSC Adv 6(99):96527-96530. https://doi. org/10.1039/C6RA20163F

53. Costa A, Costa ER, Silva ALP, Tanaka AA, de Jesus GJ (2018) Theoretical study of the effects of substituents $(\mathrm{F}, \mathrm{Cl}, \mathrm{Br}, \mathrm{CH} 3$, and $\mathrm{CN}$ ) on the aromaticity of borazine. J Mol Model 24(1):34. https:// doi.org/10.1007/s00894-017-3555-x

54. Rosenthal J, Schuster DI (2003) The anomalous reactivity of fluorobenzene in electrophilic aromatic substitution and related phenomena. J Chem Educ 80(6):679. https://doi.org/10.1021/ ed080p679

Publisher's note Springer Nature remains neutral with regard to jurisdictional claims in published maps and institutional affiliations. 